\title{
Research on the Hot Rolling of Blanks on Oval and Circle Dies
}

\author{
P.I. Zolotukhin ${ }^{1}$, I.M. Volodin ${ }^{1}$, A.I. Volodin ${ }^{1}$ \\ ${ }^{1}$ Lipetsk state technical University, Russia \\ ${ }^{1}$ Affiliation of the first author \\ ${ }^{2}$ Affiliation of the second author \\ ${ }^{3}$ Affiliation of the third author \\ *Corresponding author E-mail:
}

\begin{abstract}
The results of the blanks hot rolling modeling in the «QForm 3D» program and the research on the deformed shape (DS) of metal by grid method are presented. Comparison of data obtained by different methods is made when analyzing the rolling of blanks for two passes using the circle-oval-circle scheme.
\end{abstract}

Keywords: Sector-stamps, calibers, shaping, computer modeling, contact surface, deformation force

\section{Introduction}

Rolling process consists in getting blanks in the streams of sectors - stamps mounted on rolls, rotating towards each other. Roller is mainly used for preliminary blanks shaping $[1,2]$, used for subsequent hot forging [3].

When preparing the production of new forgings, the efficiency of the rolling technology is evaluated by analyzing the accuracy of the resulting semi-finished products $[2,4]$ and the operating conditions of the process equipment $[5,6,7]$. The design methods by V.K. Smirnov and his co-workers are now the most used at Russian engineering enterprises. The technique is based on the use of formulas obtained from the results of a numerical solution of the theoretical plastic deformation of metals problem by the variation principle of a full power minimum.

In recent years, a lot of software packages have been created for computer modeling of plastic deformation of metals using finite element method. One of these programs is the «QForm 3D» package, that allows modeling the hot volume deformation of blanks with a rotating tool.

\footnotetext{
${ }^{*}$ This research was financially supported by Ministry of Science and Education of Russia (project No. 11.9505.2017/8.9)

In this work, a comparison is made between the results of calculating the blanks shaping and the deformation force during rolling in two passes using the circle-oval-circle scheme according to the method of V.K. Smirnov with the results of modeling in the program «QForm 3D». We also compared the calculation of the metal DS characteristics using the formulas obtained by the authors, with the results of computer simulation.
}

\section{Materials and methods}

According to the circle-oval-circle scheme (Image 1) the deformation center for rolls is uniquely characterized by the following independent dimensionless parameters [8]: $A=D^{*} / H_{1}$ given diameter of rolls; $1 / \eta=H_{0} / H_{1}$ - compression ratio; $a_{k}=B_{k} / H_{1}$ - the axes ratio of the die in question; $a_{0}=H_{0} / B_{0}$ - the ratio of the blank sides in the previous die of the rolling process; $\delta_{1}=B_{1} / B_{k}$ - the degree of the given die filling by the metal; $\delta_{0}=H_{0} / H_{0}^{\prime}$ - the degree of filling the previous die in the rolling process by the metal.

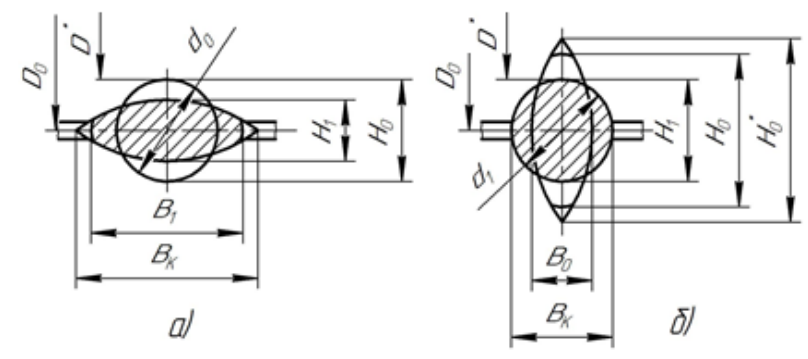

Image 1. Rolling scheme: a - circle-oval; b - oval-circle

The blanks rolling was examined according to the circle-ovalcircle scheme on the conical forging rollers, C1334 model. There is the initial data for the design of technology: the dimensions of the rolled blank (Image 2, second transition); deformable steel mark- steel 45 ; temperature of blank heating $1200^{\circ} \mathrm{C}$; distance 
The initial blank

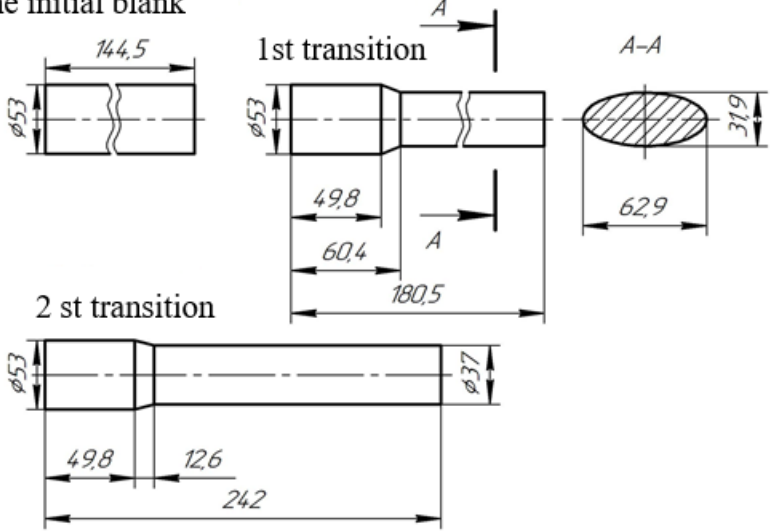

Image 2. Transitions of blanks rolling on C1334 model rollers

between roll axes $D_{0}=250 \mathrm{~mm}$; rotation speed of rolls $65 \mathrm{rpm}$; degree of oval-die filling $\delta_{1}=0,8$; degree of circle-die filling $\delta_{1}=1,0$; value of the friction rate $\psi=0,75$.

The transverse and longitudinal dies dimensions were calculated according to the blanks dimensions (Image 2), taking into account the lead of the metal and the spring of the rolls. Volumetric models of sectors - stamps and blanks were created using the graphic computer program "KOMPAS 3D".

The tool and blanks models were exported to the «QForm 3D» program and the shape change and characteristics of the metal DS during roll-rolling was consistently calculated according to the circle-oval schemes (Image 3) and the oval-circle ones. The initial data for computer modeling were the same as in the calculations by the method of V.K. Smirnov.

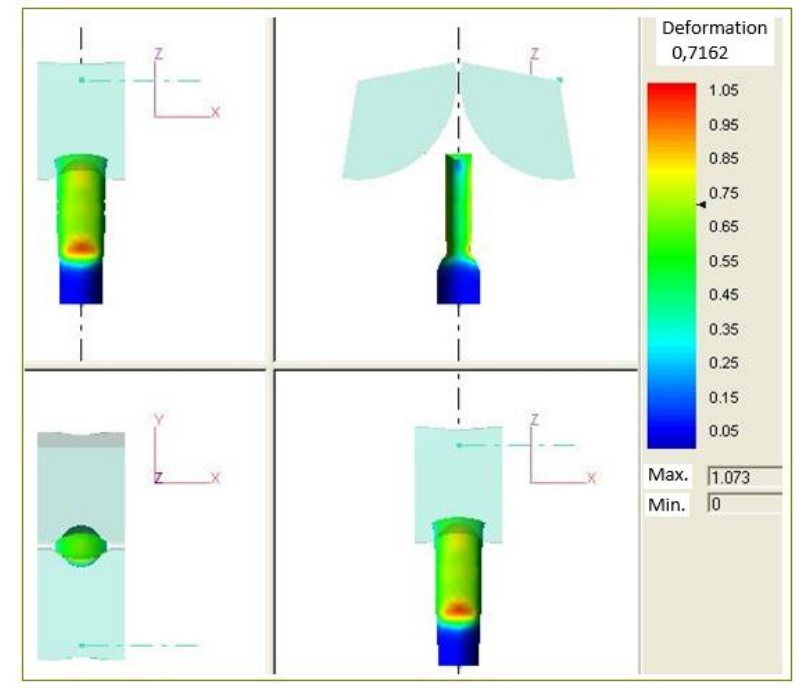

Image 3. Computer modeling of deformation and metal DS during rolling by the circle-oval scheme (1 st transition)

\section{Results and discussion}

Image 3 shows the position of the blanks and tool at the final stage of deformation, i.e. when the blank came out of the dies of the rotating sectors - stamps. The total length of the blanks after the first junction is $186.8 \mathrm{~mm}$. Thus, computer modeling predicts greater stretching than the calculation by the method of V.K. Smirnov (length $180.5 \mathrm{~mm}$ in Image 2). The relative deviation in the blank length after the first transition was $3.5 \%$.

According to the results of modeling after the second transition the blank length is $251.2 \mathrm{~mm}$, and its length by the method of V.K Smirnov - $242.0 \mathrm{~mm}$ (Image 2). The relative deviation in the blank length after two transitions was $3.8 \%$.
Using the «QForm 3D» package for the technology in question (Image 2), the local characteristics of the metal DS-strain intensity $\varepsilon_{u}$ are calculated. The total value $\varepsilon_{u}$ was determined for two junctions for two different particles on the blank surface in the middle part along the crimped section length (here the rolling process is steady). The first particle of the first pass was on the symmetry line of the blank contact surface (the upper left window in Image 3). This particle deformation intensity $\varepsilon_{u \kappa}^{(1)} \approx 0,72$ (shown by an arrow on the scale in Image 3 ). Before the second pass, the blank is tilted $90^{\circ}$ around its axis and therefore the first particle will go over to the symmetry line of the blank tool-free side. For this pass $\varepsilon_{u c}^{(1)} \approx 0,59$. Total deformation of the first particle $\varepsilon_{u}^{(1)}=\varepsilon_{u \kappa}^{(1)}+\varepsilon_{u c}^{(1)} \approx 1,31$.

The second particle was also on the line of symmetry and passed from the free lateral surface in the first pass (the right upper window in Image 3 , deformation $\varepsilon_{u c}^{(2)} \approx 0,42$ ) to the contact surface in the second pass (deformation $\varepsilon_{u \kappa}^{(2)} \approx 0,78$ ). The total deformation of this particle $\varepsilon_{u}^{(2)}=\varepsilon_{u c}^{(2)}+\varepsilon_{u \kappa}^{(2)} \approx 1,20$.

The deformation intensity $\varepsilon_{u}$ in the rolling process under consideration was also determined depending on the dimensionless parameters of the deformation focus by the regression equations given in Table. 1. These equations were obtained by P.I. Zolotukhin by approximating the results of a metal DS characteristics study by the experimental-calculated method of coordinate grids. The planned experiment to study DS by the method of grids on the blanks surface for stationary rolling in dies is made in laboratory conditions. In the experiments, samples made of a lead-antimony alloy were used.

The regression equations (presented in Table 1) are statistically significant and have multiple correlation coefficients sufficiently close to unity $(R=0,68-0,96)$.

The deformation center parameters for the circle scheme and the results of calculation using the regression equations for the first and second metal particles in the first transition $A=6,92$;

$1 / \eta=1,66 \quad ; \quad a_{\kappa}=2,47 \quad ; \quad \varepsilon_{u \kappa}^{(1)} \approx 0,56 \quad ;$ $\varepsilon_{u c}^{(2)} \approx 0,55$. The parameters for the oval-circle scheme and the results of calculation by equations for the same particles in the second transition: $A=5,82 ; 1 / \eta=1,70$; $a_{0}=1,97 ; \delta_{0}=0,8 ; \varepsilon_{u \kappa}^{(2)} \approx 0,58 ; \varepsilon_{u c}^{(1)} \approx 0,67$. Table 1

The regression equations for calculating the deformation intensity $\varepsilon_{u c}$ on the lines of free lateral symmetry $\left(\varepsilon_{u c}\right)$ and blank contact $\left(\boldsymbol{E}_{u c}\right)$ surfaces

\begin{tabular}{|l|l|}
\hline $\begin{array}{l}\text { Rolling } \\
\text { scheme }\end{array}$ & Regression equations \\
\hline $\begin{array}{l}\text { Circle } \\
\text { oval }\end{array}$ & $\varepsilon_{u c}=1,745 \cdot(A-1)^{-0,134} \cdot\left(a_{\kappa}-1\right)^{-0,802} \cdot(1 / \eta-1)^{1,482}$ \\
\cline { 2 - 3 } & $\varepsilon_{u \kappa}=1,276 \cdot(A-1)^{-0,277} \cdot\left(a_{\kappa}-1\right)^{-0,072} \cdot(1 / \eta-1)^{0,722}$ \\
\hline \multirow{2}{*}{$\begin{array}{l}\text { Oval } \\
\text { circle }\end{array}$} & $\varepsilon_{u c}=1,205 \cdot(A-1)^{-0,252} \cdot\left(a_{0}-1\right)^{-0,154} \cdot(1 / \eta-1)^{0,595} \cdot\left(\delta_{0}\right)^{-0,108}$ \\
\cline { 2 - 3 } & $\varepsilon_{u \kappa}=0,984 \cdot(A-1)^{-0,243} \cdot\left(a_{0}-1\right)^{-0,324} \cdot(1 / \eta-1)^{0,586} \cdot\left(\delta_{0}\right)^{-0,253}$ \\
\hline
\end{tabular}


Total deformations for the first and second particles: $\varepsilon_{u}^{(1)}=0,56+0,67=1,23$

$\varepsilon_{u}^{(2)}=0,55+0,58=1,13$.

Thus, the relative deviation in the deformation intensity calculation $\varepsilon_{u}$ for two passes on the regression equations from computer simulation for the first particle was approximately $6.5 \%$, for the second $-6.2 \%$.

The parameters of the blanks rolling technology for two circleoval-circle transitions on the forging ARWS-2 rollers (Figure 4), introduced at the PJSC «KAMAZ» smithy factory, were also compared with the simulation results in the «QForm 3D» program. The design was carried out in relation to the following conditions: the nominal interaxle distance of the rolls was $560 \mathrm{~mm}$; rotation speed of rolls - $52 \mathrm{rpm}$; grade steel deformed blanks - 40X; temperature of heating $-1150^{\circ} \mathrm{C}$; the pause before the first pass and between the passes is 5 seconds.

Based on the simulation results, the total length of the blank after the first pass is $219.5 \mathrm{~mm}$. The actual length is $206.5 \mathrm{~mm}$ (Image 4), i.e. the difference is $5.9 \%$.

Comparison of the rolled blank lengths after two passes showed a deviation of $5.1 \%$ (length at modeling - $262.3 \mathrm{~mm}$, actual length $248.8 \mathrm{~mm}$ (Image 4)).

The authors' experience has shown that the difference in the results of metal shaping modeling during rolling in oval- and circledies for two junctions and actual blanks lengths is about 3-6\%.
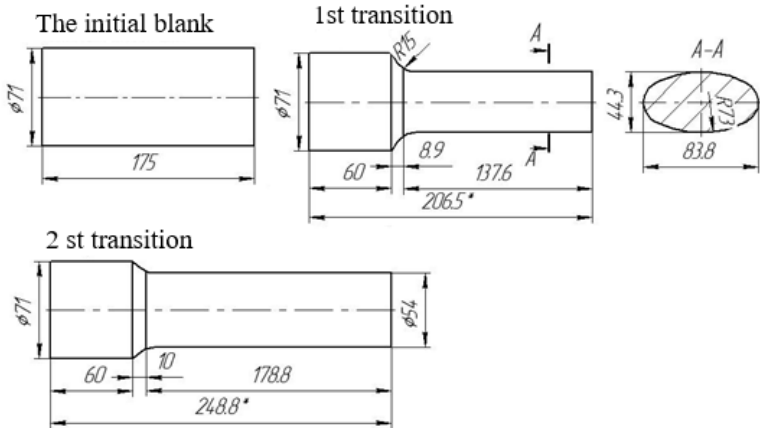

Image 4. Transitions of blanks rolling on ARWS-2 rollers

Modeling in the program «QForm 3D» determined the contact surface area for the first pass of the rolling (Image 4). This is an important parameter, on which the deformation force calculation depends to a large extent. Measurement of the contact area received in "QForm 3D" was carried out in the "KOMPAS 3D" program. Based on the simulation results, the contact area is approximately $4950 \mathrm{~mm} 2$. Calculation by the method of V.K. Smirnov gives an area equal to $5300 \mathrm{~mm} 2$, i.e. the difference is $6.6 \%$.

In the «QForm 3D» program, the deformation force $(P)$ was calculated for the first round pass in a circle-oval scheme (Image 4). The program makes it possible to fix the schedule for changing $\mathrm{P}$ in the rolling process. The maximum deformation force was approximately $600 \mathrm{kN}$. Calculation by the method of V.K. Smirnov gives $\mathrm{P} \approx 780 \mathrm{kN}$, i.e. The difference with modeling is $23 \%$. In both cases, the deformation resistance for grade $40 \mathrm{X}$ steel was determined depending on the temperature, degree and deformation speed according to literature data.

A sufficiently large difference in the values of the force $P$ from the computer simulation results and the method by V.K. Smirnov can be explained as follows. It is known that the calculation of characteristics of the stressed metal state (namely, the normal stresses on the contact surface determines the value of $\mathrm{P}$ ) for a bulk metal flow is one of the most complex problems in the continuum mechanics. Therefore, the error in computer simulation in this case is the highest (in comparison with the calculation of the deformed state characteristics and metal shaping).
It is worth noting that the methods by V.K. Smirnov has a good reputation when introducing new rolling technologies into production.

\section{Conclusions}

The experience of using the «QForm 3D» program showed a good match between the computer simulation results of metal shaping during rolling for two jumps and the results obtained in production conditions. The results of DS characteristics modeling with data obtained using the grid method also gave good agreement.

The difference in the results of deformation force determining is $20-25 \%$.

\section{References}

[1] Volodin, I.M., Zolotukhin, P.I., Karpaytis, E.P., Volodin, A.I, (2016), The influence of roll springing on the process of forgerolling of billets in calibers, Journal of Chemical Technology and Metallurgy, T. 51, No. 6, pp. 720-725.

[2] Zolotukhin, P.I., Volodin, I. M., Karpaitis, E. P., Volodin, A. I. Schmidt, A. A, (2017), Study of the spring back of calibres in material forming processes of roll forging mills. Ironmaking and Steelmaking, pp. 1-5.

[3] Telegin, I.V., Volodin, I.M., Zolotukhin, P.I, (2018), The mathematical modeling for assessing the effectiveness of hot forging extruded round in plan forgings on crank presses, International Journal of Engineering and Technology(UAE), pp. 30-34.

[4] Volodin I.M., Zolotukhin P.I., Karpaytis E.P., Volodin A.I., Romanenko E.F, (2016), Influence the process roll spring on rolling blanks in calibers, Izvestiya Yugo-Zapadnogo state Universiteta. Series: Engineering and technology, No. 4 (21), pp. 36-45.

[5] Telegin, I., Kozlov, A., Zhirkov, A, (2017), Component simulation in problems of calculated model formation of automatic machine mechanisms, MATEC Web of Conferences, p. 03016.

[6] Telegin, V., Kozlov, A, (2016), Computer realization of research into the dynamics of mechanical systems, IOP Conference Series: Materials Science and Engineering, Vol. 124, p. 012101.

[7] Telegin, V.V., Kozlov, A.M., Sakalo, V.I, (017), Solid Modeling and Dynamic Analysis of Mechanisms of Press-forging Machines, Procedia Engineering, p. 1258-1263.

[8] Smirnov V.K., Litvinov K.I., Kharitonin S.V, (1980), Hot rolling of blanks. M .: Mechanical Engineering, p. 150. 